\title{
The Phase Compensation Methods of Distributed Radar Systems
}

\author{
M. Ding, T. Sun \\ National Key Laboratory of Science and Technology on Blind Signal Processing \\ Chengdu, China
}

\begin{abstract}
The distributed radar system is a solution to detect small targets in the space. But how to effectively accumulate the received signals from different radar stations is a challenging work. The signals influenced by different time-delay, Doppler frequency and reflection coefficient will have different phases before accumulation. In this thesis, we study the phase compensation methods to eliminate the effects caused by these factors.
\end{abstract}

Keywords-distributed radar system; phase compensation; timedelay; Doppler frequency; reflection coefficient

\section{INTRODUCTION}

Nowadays, the conventional radar system is facing great challenges from stealth and small targets [1]. Though the phased-array radar system could be useful to solve the problem, its volume is usually very big, which makes it impossible to move and hard to maintain [2]. Because of these, the distributed radar system emerges.

A distributed radar system is made up of several radars that separated from each other in space, and the received signals of the radars are processed in the fusing centre in the signal level to achieve better performance of detection, tracing and imaging. In the most ideal situation, every radar station can receive signals transmitted not only from itself, but also signals from other radars. Fig.1 illustrates the composition of a distributed radar system.

The received signals of a distributed radar system composed of $\mathrm{M}$ radars are

$$
x(t)=\sum_{i=1}^{M} \sum_{j=1}^{M} \alpha_{i, j} \cdot S_{i, j}\left(t-\tau_{i, j}\right) e^{j 2 \pi\left(f_{0}+f_{d_{i, j}}\right)\left(t-\tau_{i, j}\right)}
$$

In eqn.(1), $x(t)$ represents the received signals in the data fusing centre, $S_{i, j}(t)$ represents the signal transmitted from the ith Radar and received in the jth Radar, ${ }^{\alpha_{i, j}}$ is the coefficient of reflection determined by the target, ${ }^{\tau}{ }_{i, j}$ is the time-delay, $f_{0}$ is the carrier frequency, and $f_{d_{i, j}}$ is the Doppler frequency.

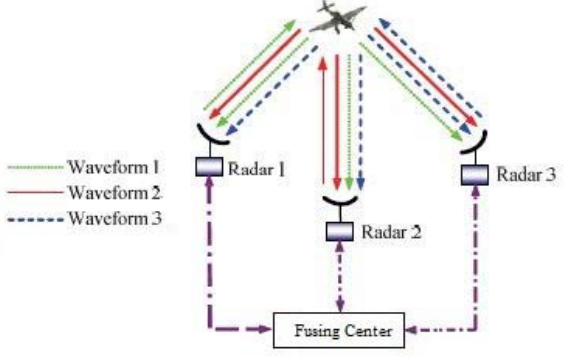

FIGURE I. DISTRIBUTED RADAR SYSTEM.

\section{PULSE ACCUMULATION IN SPACE DOMAIN}

According to the classical radar theory, pulse accumulation can increase the received signal to noise ratio (SNR).There are two kinds of accumulation methods: coherent and incoherent accumulation. If $\mathrm{M}$ pulses are accumulated, the coherent accumulation could increase the signal to noise ratio (SNR) M times, while the incoherent accumulation could increase about $\sqrt{M}$ times [3].Comparing with the conventional radar systems, the distributed radar systems could carry out the accumulation not only in the time domain, but also in the space domain. Because the accumulation in time domain has been studied by many researchers, in this thesis, we only consider the space accumulation.

\section{A. Detection Model of Space Accumulation}

The detection problem of the distributed radar system that contains $\mathrm{M}$ stations can be described as follows:

$$
\begin{aligned}
& \left\{\begin{array}{lll}
H_{0}: & r_{i}=n_{i}, & i=1,2, \ldots, M \\
H_{1}: & r_{i}=n_{i}+s_{i}, & i=1,2, \ldots, M
\end{array}\right. \\
& s_{i}=\left[s_{i}(1), s_{i}(2)\left\llcorner s_{i}(N)\right]^{H}, r_{i}=\left[r_{i}(1), r_{i}(2)\left\llcorner r_{i}(N)\right]^{H}, \quad n_{i}=\left[n_{i}(1), n_{i}(2)\left\llcorner n_{i}(N)\right]^{H}\right.\right.\right.
\end{aligned}
$$$$
H_{0} \text { represents the target doesn't exist, }{ }^{H_{1}} \text { represents the }
$$
target exists. ${ }^{s_{i}}$ and $r_{i}$ are the ${ }^{N}$ samples of each transmitted and received signal. $n_{i}$ represents the noise, which satisfies $n_{i} \sim C N\left(0, \sigma^{2}\right)$. The distribution of $\mathbf{r}=\left[r_{1}, r_{2} \mathrm{~L} r_{M}\right]$ can be described as follows,

$$
\begin{aligned}
& f_{r_{1}, r_{2} \ldots r_{M}}\left(r_{1}, r_{2} \ldots r_{M} \mid H_{0}\right)=\frac{1}{\pi^{M N} \sigma^{2 M N}} \exp \left(-\frac{1}{\sigma^{2}} \sum_{i=1}^{M} r_{i}^{H} r_{i}\right) \\
& f_{r_{1}, r_{2} \ldots r_{M}}\left(r_{1}, r_{2} \ldots r_{M} \mid H_{1}\right)=\frac{1}{\pi^{M N} \sigma^{2 M N}} \exp \left(-\frac{1}{\sigma^{2}} \sum_{i=1}^{M}\left(r_{i}-s_{i}\right)^{H}\left(r_{i}-s_{i}\right)\right)
\end{aligned}
$$


Under the Neyman-Pearson criterion, the best detector is the likelihood ratio test:

$$
\Lambda=\frac{f_{r_{1}, r_{2} \ldots r_{M}}\left(r_{1}, r_{2} \ldots r_{M} \mid H_{1}\right)}{f_{r_{1}, r_{2} \ldots r_{M}}\left(r_{1}, r_{2} \ldots r_{M} \mid H_{0}\right)}
$$

If the phases of the signals received by different radars are compensated accurately, we can accumulate them coherently. In this case, the false alert probability and detection probability can be calculated:

$$
P_{f a}=Q\left(\gamma / \sqrt{\sigma^{2} \sum_{i=1}^{M} E_{i} / 2}\right), \quad P_{D}=Q\left(\left(\gamma-\sum_{i=1}^{M} E_{i}\right) / \sqrt{\sigma^{2} \sum_{i=1}^{M} E_{i} / 2}\right)
$$

In eqn.(6), $E_{i}$ is the power of each transmitted signal, $\gamma_{\text {is }}$ the detection threshold. If the phase can't be compensated accurately, we must accumulate them incoherently. We can derive the false alert probability and detection probability as follows.

$$
P_{f a}=\frac{1}{2}\left(1-\operatorname{erf}\left(\frac{\gamma}{\sqrt{\sigma^{2} \sum_{i=1}^{M} E_{i}}}\right)\right), P_{D}=\frac{1}{2}\left(1-\operatorname{erf}\left(\frac{\gamma-\sum_{i=1}^{M} E_{i}}{\sqrt{\frac{\sigma^{2}}{2} \sum_{i=1}^{M} E_{i}}}\right)\right)
$$

\section{B. Simulations of Detection Ability}

We set the false alert probability $P_{f a}=10^{-5}$, the detection ability is illustrated in fig.2. The solid line shows the performance of coherent accumulation of the distributed radar system, while the dotted line shows the incoherent accumulation.

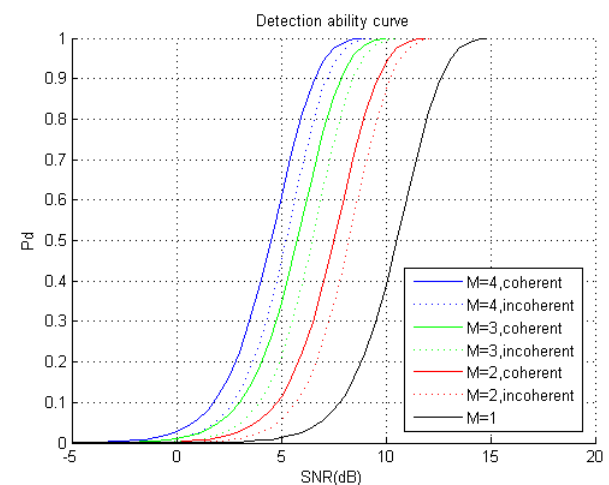

FIGURE II. DETECTION ABILITY OF DISTRIBUTED RADAR SYSTEM.

It clearly indicates that, the detection probability increases along with the amount of radars, and the coherent accumulation has a better performance over the incoherent accumulation.

\section{COMPENSATION OF TIME-DELAY AND DOPPLER FREQUENCY}

Though the coherent accumulation's superiority is obvious, the signals' phases could be different from each other due to many factors. In the worst situation, if two signals' phase difference is $\pi$, the amplitude after accumulation is 0 , which means the accumulation would make no sense. So the compensation of the phases is necessary before the accumulation.

\section{A. Compensation of Time-Delay}

Once the signals are received, we use the coherent reference signals to demodulate them. The signals after demodulation are:

$$
x^{\prime}(t)=\sum_{i=1}^{M} \sum_{j=1}^{M} \alpha_{i, j} \cdot S_{i, j}\left(t-\tau_{i, j}\right) e^{j 2 \pi\left(f_{0}+f_{d_{i, j}}\right)\left(t-\tau_{i, j}\right)} \cdot e^{-j 2 \pi f_{0} t}
$$

The time-delay caused by the different distances from radar to target not only results in different range gates after matchfilter, but also introduces a phase factor $e^{-j 2 \pi f_{0} \tau_{i, j}}$ that differs from each other, which will cause huge impact on the coherent accumulation.

We assume that all the radars have focused on the same detection unit in the space, then the time-delay's influence could be compensated by the methods below.

We can convert the coordinates of the detection unit and radar stations into a common coordinate system, then the distances from the radars to the detection unit can be calculated easily.

If $L_{i j}$ represents the path from radar i to radar j, and we set $L_{11}$ as the reference distance, the range gate's difference is

$$
\Delta \mathbf{L}=\left[0, L_{12}-L_{11}, \mathrm{~L} L_{M M}-L_{11}\right]
$$

The phase shift caused by the phase factor $e^{-j 2 \pi f_{0} \tau_{i, j}}$ could be compensated by

$$
\boldsymbol{\beta}=\left[e^{j 2 \pi f_{0} L_{11} / c}, e^{j 2 \pi f_{0} L_{12} / c}, \mathrm{~L} e^{j 2 \pi f_{0} L_{M M} / c}\right]
$$

In eqn.(10), $c$ is the speed of light and $f_{0}$ is the carrier frequency.

\section{B. Phase Compensation Caused by Doppler Frequency}

Besides the time-delay, the Doppler frequency could influence the phases of received signals, too. In order to separate the signals in the fusing centre effectively, the orthogonal waveforms are used. In this thesis, we choose the Orthogonal Frequency Division Multiplexing-Linear Frequency Modulation waveform[4], because it is easy to design and it shows good performance of Doppler tolerance. The waveform is shown below:

$$
S_{M}(t)=\frac{1}{\sqrt{T}} \operatorname{rect}\left(\frac{t}{T}\right) e^{j 2 \pi\left(f_{0} t+\frac{1}{2} \mu t^{2}+(M-1) f_{p} t\right)}
$$

In eqn.(11), $T$ represents the pulse duration, $f_{0}$ represents the carrier frequency, ${ }^{\mu}$ represents the modulation coefficient, $f_{p}$ represents the frequency interval. Suppose the bandwidth of the signal is $B$, then $\mu=B / T$.

The Doppler frequency can cause range migration and phase shift after the match-filter, so we must modify the matchfilter to suppress the impact of Doppler frequency. The modified match-filter in time-domain is

$$
h_{i, j}(\mathrm{t})=S_{i}(-t) \cdot e^{-j 2 \pi f_{d_{i, j}} t}
$$


Because the velocity can't be known in advance, we may only have the prior knowledge of velocity range, so a set of match-filters that matches different Doppler frequencies is needed.

$$
f_{i}=i \cdot \frac{2\left(V_{\text {m }}-V_{-}\right)}{\lambda(p-1)}+\frac{2 V_{\text {in }}}{\lambda} \quad i=0,1, \cdots P-1
$$

In eqn.(13), $\boldsymbol{V}_{\mathbf{m x}}$ is the maximum velocity and $\boldsymbol{V}_{\min }$ is the minimum velocity. $\boldsymbol{\lambda}$ is the carrier's wavelength. $\boldsymbol{P}$ is the number of match-filters which depends on the search steps of the velocity.

The composition of the match-filters is illustrated in fig.3.

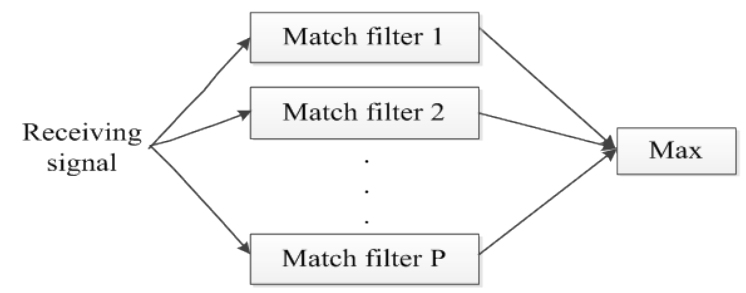

FIGURE III. COMPOSITION OF THE MATCH-FILTERS.

\section{COMPENSATION OF REFLECTION COEFFICIENT}

For a distributed radar system, the radar stations may be placed in a long distance from each other, so they observe the radars from different angles. Due to the characteristic of Radar Cross-Section(RCS), the reflection coefficient of each station may be various. Eran Fishler[5]extends the classic Swerling I model into the situation of several separated radars. In this paper, we use Eran Fishler's conclusions and give a method to compensate the phase shift caused by the reflection coefficient.

According to Eran Fishler, the reflection coefficient satisfies the complex Gaussian distribution,

$$
\alpha \sim C N(0,1)
$$

One useful method to compensate the reflection coefficient is to process the blind search algorithm. The main issue of this algorithm is its complexity. To completely compensate the phase is impossible, we can only compensate it into a small extent.

By calculation, the step length is set to $\pi / 36$. For a distributed radar system with $\mathrm{M}$ radars, the number of searches is about $5000 \mathrm{M}$, and the phase deviation can be controlled to $\boldsymbol{\pi} / 72$. The accumulation lose caused by the deviation is $0.002 \mathrm{~dB}$, which will satisfy the requirement of target detection.

However, when the blind search algorithm is conducted in a low signal to noise ratio(SNR) situation, it might occur false alert because we traverse almost all the phases from 0 to $2 \pi$.

Fig.4 illustrates the situation that noise is compensated as a target.

The false alert is set to $\mathbf{1 0}^{\mathbf{4}}$, and the detection probability is 0.95.According to eqn.(6), we can derive the required signal to noise ratio(SNR) is $11.4 \mathrm{~dB}$. One of the sampling points' amplitude is more than $11.4 \mathrm{~dB}$ over the average noise.
According to the detection method, we predict there is a target in the detection unit, but actually the target doesn't exist at all.

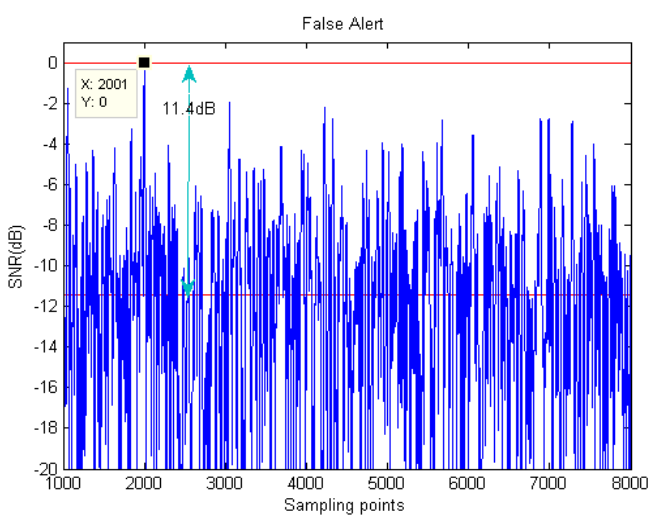

FIGURE IV. FALSE ALERT OF NOISE.

In order to solve this, we design a twice compensation algorithm to reduce the possibility of false alert.

For a target satisfies Swerling I model, the reflection coefficient can be considered to be same from one pulse to another, while for the white noise, its phase is random all the time. So, when we conduct the phase compensation, the compensated phases should be the same for a target, but for white noise, the phases compensated almost can't be the same. So we carry twice compensation algorithm and record the compensated phases. If their difference is in a small range, we can predict that the target exists.

\section{SIMULATIONS AND ANALYSIS}

We use the Monte Carlo method to test the algorithm, the parameters used are illustrated in table 1.

TABLE I. THE PARAMETERS OF THE MONTE CARLO TESTING.

\begin{tabular}{|c|c|c|c|}
\hline $\begin{array}{l}\text { Number of } \\
\text { stations } \boldsymbol{M}\end{array}$ & $\mathbf{3}$ & $\begin{array}{c}\text { Carrier frequency } \\
\boldsymbol{f}_{\mathbf{0}}\end{array}$ & $\mathbf{2 . 4 G H z}$ \\
\hline Pulse width $T$ & $10 \mathrm{~ms}$ & Bandwidth $B$ & $5 \mathrm{MHz}$ \\
\hline $\begin{array}{l}\text { Frequency } \\
\text { interval } f_{p}\end{array}$ & $50 \mathrm{MHz}$ & $\begin{array}{c}\text { Probability of false } \\
\text { alert } P_{f a}\end{array}$ & $10^{-5}$ \\
\hline $\begin{array}{l}\text { Velocity of target to each } \\
\text { station } V\end{array}$ & \begin{tabular}{c}
$-500 \mathrm{~m} / \mathrm{s}, 1000 \mathrm{~m} / \mathrm{s}, 800 \mathrm{~m} / \mathrm{s}$ \\
\hline
\end{tabular} \\
\hline
\end{tabular}

The Monte Carlo testing's result is in fig 5. We can clearly see that the twice compensation algorithm's performance is close to the theoretical value. For a distributed radar system that has three radar stations, we derive its gain of accumulation is $4.7 \mathrm{~dB}$ over the single radar from the Monte Carlo testing, which is accord with the radar theory. 


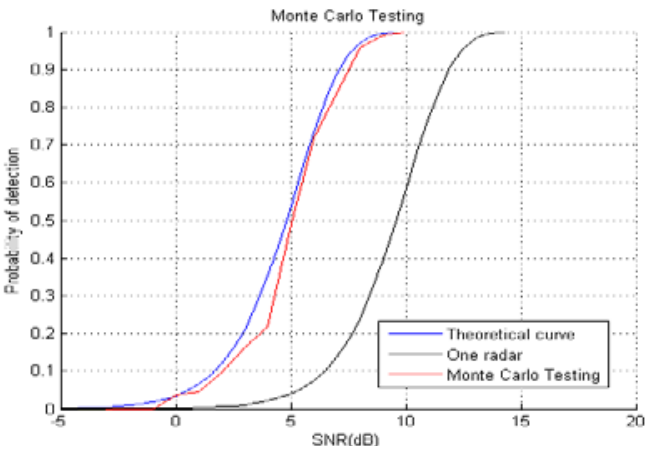

FIGURE V. MONTE CARLO TESTING.

\section{REFERENCES}

[1] Mingjun Yao, Weiming Yuan, Wenge Xing, Polyphase Orthogonal Code Design Based on Hybrid Optimization Algorithm. Modern Radar, 29(7), pp.55-60, 2007.

[2] Shuai Chang, Hong $\mathrm{Li}$, Changhui $\mathrm{Xu}$, MIMO radar detection performance analysis. Electronic Design Engineering, 19(24), pp.90-93, 2011.

[3] Mark A.Richards, Fundamentals of Radar Signal Processing. Publishing House of Electronics Industry: Beijing, pp.24-26, 2008.

[4] Deng Hai, Polyphase code design for orthogonal netted radar systems. Transactions on Signal Processing, 52(11), pp.3126 — 3135, 2004.

[5] Eran Fishler, Alex Haimovich, Rick Blum, Len Cimini, Spatial diversity in radars-models and detection performance. IEEE Transactions on Signal Processing, 54(3), pp.823-838, 2006. 